\title{
The Efficacy of Therapeutic Angiogenesis Using Basic Fibroblast Growth Factor in Patients With Coronary Artery Disease: A Double-Blind, Placebo-Controlled Study
}

Hossein Ahmadi ${ }^{*}$, Armita Mahdavi ${ }^{5}$, Morteza Daliri², Mohammad Imani ${ }^{3}$, Abbasali Karimi $^{1}$, Abbas Salehi Omran ${ }^{1}$, Mehdi Najafi ${ }^{4}$, Mohsen Ahmadi ${ }^{5}$, Farhad Fathi ${ }^{5}$, Pegah Bikdeli ${ }^{5}$, Payam Mohammadinejad 5

\footnotetext{
${ }^{1}$ Department of Cardiac Surgery, Tehran Heart Center, Tehran University of Medical Sciences, Tehran, Iran. ${ }^{2}$ Department of Animal and Marine Biotechnology, National Institute of Genetic Engineering and Biotechnology, Tehran, Iran. ${ }^{3}$ Iran Polymer and Petrochemical Institute, Tehran, Iran. ${ }^{4}$ Department of Anesthesiology, Tehran Heart Center, Tehran University of Medical Sciences, Tehran, Iran. ${ }^{5}$ Clinical Research Center, Tehran Heart Center, Tehran University of Medical Sciences, Tehran, Iran.
}

First Published online March 30, 2016

\begin{abstract}
Background and Objectives: Complete revascularization is not possible in up to $37 \%$ of patients with coronary artery disease (CAD). Therapeutic angiogenesis may be considered as an option in the management of these patients. The aim of the present study was to evaluate the effectiveness and safety of therapeutic angiogenesis using basic fibroblast growth factor (FGF) in patients with CAD.

Methods: In this double-blind, placebo-controlled study, 18 patients with a severe diffuse atherosclerotic disease along the left anterior descending (LAD) artery who were a coronary artery bypass grafting (CABG) candidate with at least one graftable coronary artery and the presence of ischemia and viable areas along the LAD were enrolled. The patients were randomized into two groups to either undergo CABG and simultaneous FGF-2 therapy (bFGF group) or CABG without FGF-2 therapy (control group). During the CABG procedure in bFGF group, FGF-2/ alginate-heparin-sepharose microcapsules, each contains 100 mcg FGF-2, were implanted in the subepicardial layer of the diffusely defective LAD territory via 2-3 mm stab incisions. Seven patients in each group were followed up for a period of 24 months.
\end{abstract}

Findings: The result of left ventricular evaluation with echocardiography and perfusion scans showed significant improvement in FGF-2-receiving group with no significant change in controls, 3 and 6 months after the intervention. New York Heart Association (NYHA) class was significantly lower in the intervention group (1.43 \pm 0.535 vs. $2.57 \pm 0.535, P=0.002$ ), In addition, intervention group remained free of angina 24 months after the intervention while three patients in the control group were hospitalized due to the acute chest pain.

Conclusions: Our study revealed that FGF-2 can improve the outcomes of patients with CAD undergoing CABG, without serious adverse effects. Considering other advantages associated with protein therapy our finding may help open novel avenues to safe and cost-effective therapy of the target patients.

Keywords: Therapeutic angiogenesis, Fibroblast growth factor, Coronary artery disease, CABG, Patient outcome, Patient safety, Patient management

\section{Background and Objectives}

Coronary artery disease (CAD) imposes a high burden in Iran and it is including $50 \%$ of deaths in this country. ${ }^{1}$ Despite the recent advances in the therapeutic methods, the morbidity and mortality of CAD are increasing mostly due

*Corresponding Author: Hossein Ahmadi, Department of Cardiac Surgery, Tehran Heart Center, Tehran University of Medical Sciences, Tehran Iran. Tel: +98 21 88029675, Fax: +98 21 88029731, Email: ahmadita@ sina.tums.ac.ir to its rising prevalence. ${ }^{2}$ Some of the patients may benefit from the restoration of the blood flow, using coronary angioplasty and stents, or coronary artery bypass grafting (CABG). CAD patients who achieve complete revascularization by CABG may enjoy a higher quality of life and survival rate. ${ }^{3}$ However, the benefits of CABG are limited since complete revascularization is not possible in up to $37 \%$ of cases because of the diffuse arterial involvement or small size of the arteries. ${ }^{3}$ Stimulation of the neovas- 
cularization as a biological bypass of the atherosclerotic vessels may improve the symptoms in patients with the severe ischemic cardiac disease who cannot benefit from the conventional percutaneous coronary intervention or $\mathrm{CABG}^{2}$ Thus far, numerous inducers of angiogenesis have been identified, including the members of the vascular endothelial growth factor (VEGF), platelet-derived growth factor (PDGF), ${ }^{4}$ transforming growth factor- $\alpha$ and $-\beta$ (TGF- $\alpha$ and $-\beta),{ }^{5}$ chemokines, interleukins, and the members of the fibroblast growth factor (FGF) family. ${ }^{6}$ Studies reported that basic FGF, also known as FGF-2 have a strong affinity for the extracellular matrix and basal lamina and also paracrine, autocrine and intracellular modes of action. ${ }^{7}$ It is reported that FGF-2 may improve the survival rate in patients with the ischemic heart disease if it is administered before, during or after the injury. ${ }^{8}$ Furthermore, exogenous administration of FGF in different animal models of chronic myocardial ischemia or acute infarction showed increased myocardial perfusion and preserved left ventricular function. ${ }^{9,10}$ In this double-blind, placebo-controlled study we evaluated the effectiveness and safety of angiogenic protein therapy by implantation of sustained-release alginate-heparin-sepharose microspheres capsules of FGF-2 in the ischemic myocardium of patients who underwent CABG.

\section{Methods}

Patient Selection

Eighteen patients who were eligible for CABG were included in this study, which was performed at the Tehran Heart Center (Tehran, Iran) between February 2007 and July 2009. The inclusion criteria consisted of severe diffuse atherosclerotic disease along the left anterior descending ( $L A D$ ) artery, being a CABG candidate with at least one graftable coronary artery, and the presence of ischemia and viable areas along the LAD. The patients were excluded if there were a need for concomitant valve repair or replacement, malignant arrhythmia, history of malignancy, hemangioma, or diabetic retinopathy, renal insufficiency (serum creatinine $>3 \mathrm{mg} / \mathrm{dL}$ ), abnormal liver function tests, and an estimated post-operation mortality risk higher than $25 \%$. Eighteen patients were enrolled sequentially, randomized into 2 groups to either undergo CABG and simultaneous FGF-2 therapy or CABG without FGF-2 therapy. For each patient, a questionnaire containing demographic information and detailed medical history was completed as well.

Preparation of FGF-2/Alginate-Heparin-Sepharose Microcapsules

FGF-2 was purchased from PeproTech (Princeton Busi- ness Park, Rocky Hill, USA), and heparin-sepharose and other chemicals from Sigma-Aldrich (Seelze, Germany). The microcapsules were prepared as per the method of Edelman et $\mathrm{al}^{11}$ and Selke et $\mathrm{al}^{12}$ with little modifications. Briefly, $1 \mathrm{~mL}$ of heparin-sepharose suspension (33.3 mg) was sterilized under ultraviolet light for $\mathbf{3 0}$ minutes in a disposable sterile petri dish (35 mm). Except heparin-sepharose suspension, other solutions were sterilized by 0.22 disposable filters. Sepharose suspension was washed 3 times with sterile water (1:1) and mixed with $1 \mathrm{~mL}$ of filter-sterilized sodium alginate $(1.2 \%, w / v)$. After mixing, the slurry was dropped through a fine needle into a beaker containing $500 \mathrm{~mL}$ hardening solution of $\mathrm{CaCl}_{2}(1.5 \%$, w/v) with continuous mixing using a magnetic stirrer. Immediately, formed microcapsules were incubated in the $\mathrm{CaCl}_{2}$ solution for 5 minutes under gentle mixing, and then for 10 minutes without mixing. The microcapsules were washed three times with sterile water. Afterward, 25 to 30 microcapsules were kept in a microfuge tube containing $0.9 \%$ $\mathrm{NaCl}, 1 \mathrm{mM} \mathrm{CaCl}, 0.05 \%$ gelatin and $100 \mu \mathrm{g} \mathrm{FGF}$. Microfuge tube was kept on shaker at $4^{\circ} \mathrm{C}$ for $16-20$ hours. Microcapsules could be stored at $4^{\circ} \mathrm{C}$ for 3 months in a solution of $150 \mathrm{mM} \mathrm{NaCl}$ and $1 \mathrm{mM} \mathrm{CaCl}_{2}$. The procedures were conducted under laminar flow to avoid any contamination. Furthermore, after the completion of each preparation, several microcapsules were evaluated for contamination using the culture media to confirm their sterility. The microcapsules were sent under the sterile condition to the operating room at the Tehran Heart Center on the day of the surgical procedure.

Surgical Procedure and Implantation of FGF-2 Containing Microcapsules

Standard hematologic laboratory tests and evaluation of cardiac enzymes for assessment of myocardial ischemia, evaluation of serum creatinine level, liver function tests, urinalysis, baseline electrocardiography, LV echocardiography, dobutamine stress echocardiography (DSE) and myocardial perfusion scintigraphy were performed on all patients as indicated before undergoing the cardiac surgery. All patients were operated on by a cardiac surgery team according to the standardized surgical protocol. Following sternotomy, the patients were heparinized and cannulated. Cardiopulmonary bypass (CPB) was established. Then patients were cooled down to $28^{\circ}-30^{\circ} \mathrm{C}$. After cross-clamping of the aorta, 0.8 to $1.0 \mathrm{~L}$ of cold blood cardioplegic solution was infused into the aortic root with a mean pressure of 50 to $70 \mathrm{~mm} \mathrm{Hg}$. At first the distal coronary anastomoses were performed. Systemic rewarming was started before the final distal anastomoses were completed. Subsequently, 10 FGF-2/alginate-heparin-sephar- 
ose microcapsules each contains 100 mcg FGF-2 were implanted in the subepicardial layer of the diffusely defective LAD territory via 2-3 mm stab incisions. ${ }^{3}$ After the implantation, the epicardial incisions were sutured with 5-0 polypropylene. Afterward, proximal anastomoses were performed, the aorta was unclamped and the patients were separated from CPB and the chest was closed normally.

\section{Follow up}

After surgery, patients were transferred to the intensive care unit (ICU) for precise monitoring of hemodynamic status and arrhythmia. Indicated blood tests were also performed. After 24-48 hours, patients were discharged from the ICU to the ward if their hemodynamic status was stable. Thereafter, the operation complications were investigated in the patients. Complete blood counts, liver function tests, serum chemistries, urinalysis, and electrocardiography were performed on the seventh day, third month and sixth month after the operation. Besides the clinical investigations and blood test evaluations, electrocardiography, New York Heart Association (NYHA) class evaluation and myocardial perfusion scintigraphy were repeated at the 6th month of the follow-up.

In the second phase of this study (for the long term follow up), between September 2008 and February 2010, all patients were contacted by the investigators to assess clinical events (mortality, myocardial infarction, recurrent angina, ICU admission, or any subsequent complications) Perfusion scan and echocardiography were performed in all patients 6 months after surgery. Besides, 18 to 24 months after surgery, they were asked by phone conducted interviews for their clinical symptoms, morbidity, mortality or NYHA functional class.

\section{Statistical Analysis}

All analyzes were performed by operators blinded to al clinical and other functional data using SPSS version 11.5 software. Data are presented as mean \pm standard deviation (SD). Paired samples $t$ test and independent samples $t$ test were used as appropriate. Fisher exact test was performed to compare clinical variables between groups. A $P$ value less than 0.05 was considered significant.

\section{Ethical Issues}

This study was approved by the Ethics Committee of Tehran University of Medical Sciences. The detailed process of the study, including the diagnostic and therapeutic procedures was explained to the patients and the informed consent to participate in the study was obtained from all target patients.

\section{Results}

Patients' Characteristics

Eighteen patients who met the inclusion and exclusion criteria were divided into two groups. Ten patients were allocated to bFGF group and 8 patients were allocated to control group. During the process of this study, 2 patients of the bFGF group were excluded, because they refused to participate in the follow up evaluations. Also, 2 patients (one from each group) were withdrawn from the study since they could not be located for follow up. Finally, 7 patients in each group were investigated till the end of this study. Patients were randomly assigned into two groups and there were no significant demographic differences between them. Preoperative characteristics of patients are summarized in Table 1. The prevalence of various risk factors and co-morbidities (diabetes, hypertension and smoking) were similar in both groups.

\section{Functional Status According to NYHA Classification}

NYHA functional class before the intervention was similar between the FGF-2 receiving group and control group $(2.29 \pm 0.488$ vs. $2.57 \pm 0.535, P=0.317)$. However, it was significantly lower in the FGF-2 group than control group 6 months after the intervention $(1.43 \pm 0.535$ vs. $2.14 \pm$ $0.378, P=0.014)$; this significant difference was also observed 24 months after the surgery $(1.43 \pm 0.535$ vs. 2.57 $\pm 0.535, P=0.002$ ).

NYHA functional class was significantly reduced in FGF2 receiving group 6 and 24 months after the intervention, comparing to the time before the surgery $(1.43 \pm 0.535$ vs. $2.29 \pm 0.488, P=0.001$, and $1.43 \pm 0.535$ vs. $2.29 \pm$ $0.488, P=0.001$, respectively). NYHA functional class did not significantly change in the control group at 6 and 24 months after the intervention, comparing to the time before the surgery $(2.14 \pm 0.378$ vs. $2.57 \pm 0.535, P=0.2$ and $2.57 \pm 5.535$ vs. $2.57 \pm 0.535, P=1$, respectively). In both groups, NYHA functional class did not change significantly from the 6th month to 24th months of follow up.

Evaluation of Left Ventricular Function With Echocardiography According to the 2 dimensional echocardiography, there

Table 1. Patients' Characteristics and Co-morbidities

\begin{tabular}{lll}
\hline & FGF-2 $(\mathbf{n}=\mathbf{7})$ & Control $(\mathbf{n}=\mathbf{7})$ \\
\hline Age $(\mathrm{y})$ & $58.29 \pm 6.57$ & $59.57 \pm 9.86$ \\
Male/female $(\mathrm{n})$ & $6 / 1$ & $6 / 1$ \\
Diabetes mellitus $(\mathrm{n})$ & 3 & 4 \\
Hypertension $(\mathrm{n})$ & 3 & 2 \\
Smoking $(\mathrm{n})$ & 3 & 3 \\
\hline
\end{tabular}


was no significant difference between the preoperative (baseline) ejection fraction of the FGF-2 and control groups ( $35 \pm 4.082$ vs. $33.14 \pm 2.410, P=0.32)$. However, after the 6 months of follow up, a significantly higher ventricular ejection fraction was observed in the FGF-2 group compared to the control group $(46.43 \pm 3.78$ vs. $30.71 \pm$ $5.345, P<0.001)$. Ventricular ejection fraction improved significantly in the FGF-2 group after the 6 months of follow up compared to its value before the intervention (35.00 \pm 4.082 vs. $46.43 \pm 3.780, P<0.001)$. In contrast, this value did not change significantly in the control group after the 6 months of follow up comparing to the time before the surgery $(30.71 \pm 5.345$ vs. $33.14 \pm 2.140, P=0.18)$.

\section{Perfusion Scans}

According to myocardial perfusion scan and uptake of radioactive material we had three types of segments as listed here; (1) normal uptake segment, (2) mild to moderate uptake segment, and (3) segment with severely decreased to absent uptake. Nine segments were defined for the LAD related area. There were improvements in perfusion scores within the FGF-2 group while this finding was not observed in the control group. This improvement included increases in the number of segments with normal uptake and reduction in the number of segments with mild to moderate or severely decreased or absent uptake $(P<0.001, P<0.001$ and $P=0.003$ respectively). We compared changes in perfusion scan between 2 groups which is summarized in Table 2. There was a significant difference between the FGF-2 treated and control group in the changes of the number of severely decreased to absent and normal uptake segments $(P=0.03, P=0.01)$. This finding was not observed before the intervention. Comparison of changes in perfusion scores between the 2 groups is shown in Figure 1.

The mean summed stress score was not significantly different between FGF-2 and control groups before the intervention ( $17.571 \pm 3.20$ vs. $17.85 \pm 6.01, P=0.138)$. However, patients in the FGF-2 group had significantly lower score, 6 months after the surgery $(5.428 \pm 7.299$ vs. $14.857 \pm 3.976, P=0.011)$. This score was significantly reduced in the FGF-2 group after 6 months of follow up
(5.428 \pm 7.299 vs. $17.571 \pm 3.20, P=0.001)$ in contrast to the control group who did not show a significantly lower score 6 months after the intervention $(14.857 \pm 3.976$ vs. $17.857 \pm 6.011, P=0.156)$.

\section{Postoperation Side Effects}

No serious adverse reactions associated with FGF-2 were observed. During the 24 months of follow up no post operative side effects (bleeding, myocardial infarction, cardiac arrest, chest pain, etc) were seen in the FGF-2 group and all cases in this group remained free of angina. However, 3 patients (42.85\%) of the control group were admitted to hospital because of the acute chest pain $(P=0.192$, Fisher exact test).

\section{Discussion}

Up to $37 \%$ of patients with ischemic heart disease, suffer from a diffuse and severe CAD that neither can undergo percutaneous coronary intervention nor surgical bypass to restore the normal blood flow. ${ }^{13}$ Therapeutic angiogenesis can be considered as an option in the management of these patients. Protein therapy poses some advantages since it can be delivered directly to the target area, its quantity can be controlled more accurately, can immediately affect the target tissue and probably causes minimum inflammatory and immune adverse effect compared to the other methods. ${ }^{14,15}$ More importantly, it does not require complicated methods of gene therapy or the exposure to foreign genetic materials with unknown longterm outcomes. ${ }^{16}$ The aim of this study was to evaluate the effectiveness and the safety of administering FGF-2 for therapeutic angiogenesis in ischemic myocardium of patients who undergo CABG. Administration of FGF-2 at the onset of ischemia is reported to reduce injury and attenuate heart enzymes. ${ }^{17,18}$ It can be a promising choice of pretreatment in CABG to reduce the ischemic injury of cross-clamp time. Nakamae et al reported that FGF-2 enhanced vascular density in necrotic iliac bone in rabbits. ${ }^{19}$ Previous studies showed the angiogenic effects of FGF2 in limb ischemia. ${ }^{20,21}$ Yanagisawa-Miwa et al improved cardiac function by intracoronary administration of FGF-2 in infarcted canine heart model; also FGF increased the

Table 2. The Result of Myocardial Perfusion Scintigraphy (Myocardial Segment Uptake Before and After Surgery in Two Groups)

\begin{tabular}{|c|c|c|c|c|c|c|c|}
\hline \multirow{2}{*}{ Uptake Segment } & \multicolumn{3}{|c|}{ FGF-2 Treated Group } & \multicolumn{3}{|c|}{ Control Group } & \multirow{2}{*}{$\begin{array}{c}\text { Changes of Perfusion } \\
\text { Scores Between Two Groups } \\
P \text { Value }\end{array}$} \\
\hline & $\begin{array}{l}\text { Before } \\
\text { Surgery }\end{array}$ & $\begin{array}{c}6 \text { Month After } \\
\text { Surgery }\end{array}$ & $P$ & $\begin{array}{l}\text { Before } \\
\text { Surgery }\end{array}$ & $\begin{array}{c}6 \text { Month After } \\
\text { Surgery }\end{array}$ & $P$ & \\
\hline Normal uptake & $3 \pm 1$ & $7.29 \pm 2.36$ & $<0.001$ & $1.71 \pm 2.43$ & $3.29 \pm 2.13$ & 0.052 & $<0.05$ \\
\hline Mild to moderate uptake & $1.43 \pm 0.53$ & $0.14 \pm 0.37$ & $<0.001$ & $2.86 \pm 2.41$ & $2.14 \pm 2.26$ & 0.283 & 0.385 \\
\hline Severe to absent uptake & $4.57 \pm 0.97$ & $1.57 \pm 2.07$ & 0.003 & $4.43 \pm 1.90$ & $3.57 \pm 1.39$ & 0.225 & $<0.05$ \\
\hline
\end{tabular}




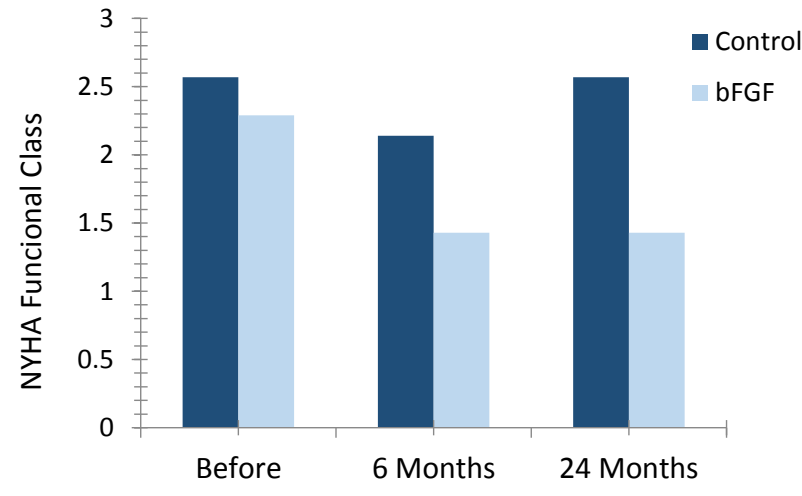

Time (Months)

Figure 1. Functional Class (NYHA) Before Operation, 6 and 24 Months After the Operation.

number of arterioles and capillaries in the infarcted area. ${ }^{18}$ Several other studies also have applied FGF-2 for inducing angiogenesis in cardiac ischemia. Scheinowitz et al administered FGF-2 via peritoneum after coronary artery occlusion in rats for a six-week period but found no sig nificant recovery in the ischemic tissue. ${ }^{22}$ This might be related to the short time of arterial occlusion or peritoneal delivery of the drug. The first trial designed to examine the safety, pharmacokinetics, and efficacy of FGF-2, in which 0-, 0.3-, 3-, or 30- $\mu \mathrm{g} / \mathrm{kg}$ doses of FGF-2 delivered intracoronary to 337 patients that were not suitable candidates for percutaneous or surgical revascularization. ${ }^{23}$ No significant differences were seen between treatment and control groups in exercise tolerance test (ETT) time assessed after 90 or 180 days. ${ }^{23}$ Angina frequency significantly reduced at 90 days, but the difference was no longer existed after 180 days. ${ }^{23}$ Ruel et al also used different doses of FGF-2 in patients with ischemic heart disease and reported a higher ejection fraction of the left ventricle and lower sum of stress perfusion found with FGF-2 group. ${ }^{24,25}$ The severity of symptoms in our population study was evaluated using NYHA Classification. It showed significant improvement in FGF group 6 and 24 months after intervention while in the control group, improvement was not significant. This finding was in agreement with the result of the study performed by Simons et al ${ }^{23}$ who reported a better physical well-being 3 and 6 months after the intervention in FGF-2 receiving group. However, this improvement was not significantly higher in the FGF-2 group compared to the control group. ${ }^{24}$ Most of the previous studies reported FGF-2 increased ventricular ejection fraction in patients receiving FGF compared to control group. ${ }^{26}$ In line with these findings, in the present study, left side ventricular ejection fraction improved significantly in FGF group 6 months after the surgery while this finding was not observed in the control group. Myocardial perfusion scan was performed 6 months after the intervention in all patients. In the FGF group, segments with impaired uptake significantly decreased while this finding was not significant in the control group.

In a phase I clinical trial by Sellke et al the result of perfusion scan 3 months after surgery of 7 patients receiving FGF showed enhanced perfusion in 3 , minimal changes in 3 and the appearance of new small defects in 1 patient. ${ }^{12}$ However, Simons et al reported no significant difference in myocardial perfusion of patients in FGF-2 group and the control group after 6 months of follow-up. ${ }^{23} \mathrm{~A}$ limitation in FGF therapy in cardiovascular patients is its relatively short serum and tissue half-life, ${ }^{26,27}$ which would cause the need of repetitive administration or higher doses. High doses of these factors can cause vasodilatation and hypotension. In addition, a long-term administration of highdose FGF can lead to membranous nephropathy. ${ }^{28}$ It was observed that endothelial cell growth factors showed a significant affinity for heparin. ${ }^{29}$ The FGF family group contains heparin/heparan sulfate binding domains. ${ }^{30}$ Heparin alginate capsules are an appropriate choice for FGF delivery, which is also used in our study. Other studies have used fibrin glue for drug delivery of growth factors, ${ }^{31-33}$ that has shown enhancing the effects of VEGF. ${ }^{31}$ Also, it can serve as a temporary matrix for the granulation tissue development. ${ }^{31}$ However, it is preferred to use recombinant human fibrinogen since it may prevent antibody reactions or possible infection transmission via human or animal derived fibrinogen. ${ }^{34,35}$ Laham et al. in 1999 showed that local perivascular implantation of heparin-alginate pellets containing 10 or $100 \mu \mathrm{g}$ of bFGF or placebo in ischemic and viable but ungraftable myocardial territories in patients undergoing CABG are safe and feasible in patients with viable myocardium that cannot be adequately revascularized. Also, reduction in the target ischemic area in the 100-mg bFGF group was shown via magnetic resonance assessment. ${ }^{3}$ Another concern regarding the use of FGF-2 is its safety due to its probable side effects, including acute severe hypotension and renal insufficiency. ${ }^{16,36}$ According to the previous studies, single local administration of FGF2 seems to be well-tolerated and safe. ${ }^{12,16,23}$ In our study, no patient showed any serious side effect. However, since 2 patients in the FGF-2 group did not participate in the follow-up evaluations after the intervention, and 2 patients, one from the FGF-2 group and another from control group could not be located till the end of the follow-up period, accurate evaluation of side effects and mortality could not be performed.

Study Limitations 
The lack of double blinded placebo controlled trial and a few findings supporting the efficacy of administering FGF2 for angiogenesis proposes in CAD patients have prevented the usage of this therapeutic method at the clinical setting. Further double blinded, placebo-controlled studies are needed to establish the benefits of this therapeutic method at the clinical setting.

\section{Conclusions}

The present study was aimed to evaluate the effectiveness and safety of therapeutic angiogenesis using basic FGF in patients with CAD. Our results revealed that FGF-2 can improve the outcomes of patients with CAD undergoing CABG, without serious adverse effects. Considering other advantages associated with protein therapy our finding may help to develop novel approaches to safe and cost-effective therapy of the target patients.

\section{Abbreviations}

(CAD): coronary artery disease; (LAD): left anterior descending; (CABG): coronary artery bypass grafting; (FGF): fibroblast growth factor; (VEGF): vascular endothelial growth factor; (PDGF): platelet-derived growth factor; (DSE): dobutamine stress echocardiography; (CPB): cardiopulmonary bypass; (ICU): intensive care unit

\section{Authors' Contributions}

The authors have made the same contributions to the research

\section{Competing Interests}

The authors declare no competing of interests.

\section{Acknowledgments}

We would like to thank the nursing, administrative and secretarial staff of the cardiology department and clinic at Tehran Heart Center for their contribution to the maintenance of our patient record without which this project would have been impossible.

\section{References}

1. Hatmi ZN, Tahvildari S, Gafarzadeh Motlag A, Sabouri Kashani A. Prevalence of coronary artery disease risk factors in Iran: a population based survey. BMC Cardiovasc Disord .2007;7:32. doi:10.1186/1471-2261-7-32.

2. Roger VL, Go AS, Lloyd-Jones DM, et al. Executive summary: heart disease and stroke statistics--2012 update: a report from the American Heart Association. Circulation. 2012;125(1):188-197. doi:10.1161/CIR.0b013e3182456d46.

3. Laham RJ, Sellke FW, Edelman ER, et al. Local perivascular delivery of basic fibroblast growth factor in patients undergoing coronary bypass surgery: results of a phase I randomized, double-blind, placebo-controlled trial. Circulation. 1999;100(18):1865-1871.

4. Kano MR, Morishita Y, Iwata C, et al. VEGF-A and FGF2 synergistically promote neoangiogenesis through enhancement of endogenous PDGF-B-PDGFRbeta signaling. J Cell Sci. 2005;118(Pt 16):3759-3768. doi:10.1242/jcs.02483.

5. Willems-Widyastuti A, Alagappan VK, Arulmani U, et al. Transforming growth factor-beta 1 induces angiogenesis in vitro via VEGF production in human airway smooth muscle cells. Indian J Biochem Biophys. 2011;48(4):262-269.

6. Presta M, Dell'Era P, Mitola S, Moroni E, Ronca R, Rusnati M. Fibroblast growth factor/fibroblast growth factor receptor system in angiogenesis. Cytokine Growth Factor Rev. 2005;16(2):159-178. doi:10.1016/j.cytogfr.2005.01.004.

7. Seghezzi G, Patel S, Ren CJ, et al. Fibroblast growth factor-2 (FGF-2) induces vascular endothelial growth factor (VEGF) expression in the endothelial cells of forming capillaries: an autocrine mechanism contributing to angiogenesis. J Cell Biol. 1998;141(7):1659-1673.

8. Kardami E, Detillieux K, Ma X, et al. Fibroblast growth factor-2 and cardioprotection. Heart Fail Rev. 2007;12(34):267-277. doi:10.1007/s10741-007-9027-0.

9. Harada K, Friedman M, Lopez JJ, et al. Vascular endothelial growth factor administration in chronic myocardial ischemia. Am J Physiol. 1996;270(5 Pt 2):H1791-1802.

10. Battler A, Scheinowitz $M$, Bor A, et al. Intracoronary injection of basic fibroblast growth factor enhances angiogenesis in infarcted swine myocardium. J Am Coll Cardiol. 1993;22(7):2001-2006.

11. Edelman ER, Nugent MA, Karnovsky MJ. Perivascular and intravenous administration of basic fibroblast growth factor: vascular and solid organ deposition. Proc Natl Acad Sci U S A. 1993;90(4):1513-1517.

12. Sellke FW, Laham RJ, Edelman ER, Pearlman JD, Simons $M$. Therapeutic angiogenesis with basic fibroblast growth factor: technique and early results. Ann Thorac Surg. 1998:65(6):1540-1544

13. Levin DC, Beckmann CF, Sos TA, Sniderman K. Incomplete myocardial reperfusion despite a patent coronary bypass: a generally unrecognized shortcoming of the surgical approach to coronary artery disease. Radiology. 1982;142(2):317-321.

14. Gal D, Weir L, Leclerc G, Pickering JG, Hogan J, Isner JM. Direct myocardial transfection in two animal models. Evaluation of parameters affecting gene expression and percutaneous gene delivery. Lab Invest. 1993;68(1):18-25.

15. Wells DJ. Improved gene transfer by direct plasmid injection associated with regeneration in mouse skeletal muscle. FEBS Lett. 1993;332(1-2):179-182.

16. Mitsos S, Katsanos K, Koletsis E, et al. Therapeutic 
angiogenesis for myocardial ischemia revisited: basic biological concepts and focus on latest clinical trials. Angiogenesis. 2012;15(1):1-22. doi:10.1007/s10456-0119240-2.

17. Jiang ZS, Padua RR, Ju $\mathrm{H}$, et al. Acute protection of ischemic heart by FGF-2: involvement of FGF-2 receptors and protein kinase C. Am J Physiol Heart Circ Physiol. 2002;282(3):H1071-1080. doi:10.1152/ ajpheart.00290.2001.

18. Yanagisawa-Miwa A, Uchida Y, Nakamura F, et al. Salvage of infarcted myocardium by angiogenic action of basic fibroblast growth factor. Science. 1992;257(5075):14011403.

19. Nakamae A, Sunagawa T, Ishida O, et al. Acceleration of surgical angiogenesis in necrotic bone with a single injection of fibroblast growth factor-2 (FGF-2). J Orthop Res. 2004;22(3):509-513. doi:10.1016/j.orthres.2003.10.001.

20. Simpson PJ, Lucchesi BR. Free radicals and myocardial ischemia and reperfusion injury. J Lab Clin Med. 1987;110(1):13-30.

21. Kloner RA, Przyklenk K, Whittaker P. Deleterious effects of oxygen radicals in ischemia/reperfusion. Resolved and unresolved issues. Circulation. 1989;80(5):1115-1127.

22. Scheinowitz M, Kotlyar AA, Zimand S, et al. Effect of basic fibroblast growth factor on left ventricular geometry in rats subjected to coronary occlusion and reperfusion. Isr Med Assoc J. 2002;4(2):109-113.

23. Simons M, Annex BH, Laham RJ, et al. Pharmacological treatment of coronary artery disease with recombinant fibroblast growth factor-2: double-blind, randomized, controlled clinical trial. Circulation. 2002;105(7):788-793.

24. Ruel M, Laham RJ, Parker JA, et al. Long-term effects of surgical angiogenic therapy with fibroblast growth factor 2 protein. J Thorac Cardiovasc Surg. 2002;124(1):28-34.

25. Ruel M, Sellke FW. Angiogenic protein therapy. Semin Thorac Cardiovasc Surg. 2003;15(3):222-235.

26. Simons M, Bonow RO, Chronos NA, et al. Clinical trials in coronary angiogenesis: issues, problems, consensus: An expert panel summary. Circulation. 2000;102(11):E73-86

27. Nakajima H, Sakakibara Y, Tambara K, et al. Therapeutic angiogenesis by the controlled release of basic fibroblast growth factor for ischemic limb and heart injury: toward safety and minimal invasiveness. J Artif Organs. 2004;7(2):58-61. doi:10.1007/s10047-004-0252-1

28. Post MJ, Laham R, Sellke FW, Simons M. Therapeutic angiogenesis in cardiology using protein formulations. Cardiovasc Res. 2001;49(3):522-531.

29. Dailey L, Ambrosetti D, Mansukhani A, Basilico C. Mechanisms underlying differential responses to FGF signaling. Cytokine Growth Factor Rev. 2005;16(2):233-247. doi:10.1016/j.cytogfr.2005.01.007.

30. Galzie Z, Kinsella AR, Smith JA. Fibroblast growth factors and their receptors. Biochem Cell Biol. 1997;75(6):669-685.

31. Wilcke I, Lohmeyer JA, Liu S, et al. VEGF(165) and bFGF protein-based therapy in a slow release system to improve angiogenesis in a bioartificial dermal substitute in vitro and in vivo. Langenbecks Arch Surg. 2007;392(3):305-314. doi:10.1007/s00423-007-0194-1

32. Kipshidze N, Chekanov V, Chawla P, et al. Angiogenesis in a patient with ischemic limb induced by intramuscular injection of vascular endothelial growth factor and fibrin platform. Tex Heart Inst J. 2000;27(2):196-200.

33. Fasol R, Schumacher B, Schlaudraff K, Hauenstein KH, Seitelberger R. Experimental use of a modified fibrin glue to induce site-directed angiogenesis from the aorta to the heart. J Thorac Cardiovasc Surg. 1994;107(6):1432-1439.

34. Prunkard D, Cottingham I, Garner I, et al. High-level expression of recombinant human fibrinogen in the milk of transgenic mice. Nat Biotechnol. 1996;14(7):867-871. doi:10.1038/nbt0796-867.

35. Butler SP, van Cott K, Subrumanian A, Gwazduaskas $\mathrm{FC}$, Velander $\mathrm{WH}$. Current progress in the production of recombinant human fibrinogen in the milk of transgenic animals. Thromb Haemost. 1997;78(1):537-542.

36. Unger EF, Goncalves L, Epstein SE, et al. Effects of a single intracoronary injection of basic fibroblast growth factor in stable angina pectoris. Am J Cardiol. 2000;85(12):14141419.

Please cite this article as:

Ahmadi $H$, Mahdavi A, Daliri M, et al. The efficacy of therapeutic angiogenesis using basic fibroblast growth factor in patients with coronary artery disease: a double-blind, placebo-controlled study. Int J Hosp Res. 2016;5(1):22-28. doi:10.15171/ijhr.2016.05. 